\title{
Pushing NRQCD to the limit
}

\author{
Chris Stewart and Roman Koniuk ${ }^{a}$

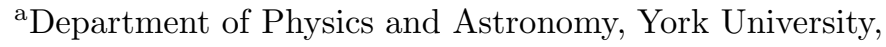 \\ 4700 Keele St., Toronto, Ontario, Canada M3J 1P3
}

Lattice NRQCD has proven successful in describing the physics of the upsilon system and $B$ mesons, though some concerns arise when it is used in simulations of charm quarks. It is certainly possible that the NRQCD expansion is not converging fast enough at this scale. We present some preliminary results on the low-mass breakdown of NRQCD, in particular the behaviour of $Q \bar{Q}$ and $Q \bar{q}$ spectra as the bare $Q$ mass is decreased well below 1 , with the aim of understanding more about the manifestation of this breakdown.

\section{Introduction}

Lattice NRQCD studies of heavy-quark systems have been, on the whole, very successful. The predicted spectra for the Upsilon, $J / \Psi, B$ and $D$ systems, amongst others, agree with the overall structure of the experimental spectra, and for the heavier systems, the agreement at finer scales, such as hyperfine splittings, is at the percent level [1].

Trottier's work on the charmonium spectrum [5] indicates that the $\mathcal{O}\left(v^{6}\right)$-improved NRQCD action decreases the hyperfine splitting significantly over the $\mathcal{O}\left(v^{4}\right)$ result, rather than taking it towards the experimental value. Our own results using similar parameters to Trottier confirm this result. This is an indication that NRQCD is not converging well for the charm quark.

Yet it is interesting to note that many successful quark model predictions of the light-quark spectrum used a non-relativistic approximation for the light-quark dynamics [2]. Here we have a highly relativistic system reasonably well described with a non-relativistic theory.

Recently, Liu et al. [3] published work on a model QCD theory known as Valence QCD. In VQCD, all z-graphs are removed, and the authors were able to make some links between NR quark models and the role of z-graphs in standard QCD. We would like to examine the behaviour of the low-mass limit of NRQCD, to examine the nature of the inevitable breakdown of the NR expansion, and see if this can also be linked to the remarkable success of NR quark models. In this report we present results for the $Q \bar{Q}$ and $Q \bar{q}$ spectra, as a function of decreasing heavy quark mass.

\section{Simulation details}

We used an $\mathcal{O}\left(v^{6}\right)$-improved NRQCD Hamiltonian,

$$
\begin{aligned}
H_{0}= & \frac{-\triangle^{(2)}}{2 M_{0}}, \\
\delta H= & -\frac{c_{1}}{8 M_{0}^{3}}\left(\triangle^{(2)}\right)^{2}+\frac{i c_{2}}{8 M_{0}^{2}}(\tilde{\triangle} \cdot \tilde{\mathbf{E}}-\tilde{\mathbf{E}} \cdot \tilde{\triangle}) \\
& -\frac{c_{3}}{8 M_{0}^{2}} \sigma \cdot(\tilde{\triangle} \times \tilde{\mathbf{E}}-\tilde{\mathbf{E}} \times \tilde{\triangle}) \\
& -\frac{c_{4}}{2 M_{0}} \sigma \cdot \tilde{\mathbf{B}}+\frac{c_{5}}{24 M_{0}} \triangle^{(4)} \\
& -\frac{c_{6}}{16 n M_{0}^{2}}\left(\triangle^{(2)}\right)^{2}-\frac{c_{7}}{8 M_{0}^{3}}\left\{\tilde{\triangle}^{(2)}, \sigma \cdot \tilde{\mathbf{B}}\right\} \\
& -\frac{3 c_{8}}{64 M_{0}^{4}}\left\{\tilde{\triangle}{ }^{(2)}, \sigma \cdot(\tilde{\triangle} \times \tilde{\mathbf{E}}-\tilde{\mathbf{E}} \times \tilde{\triangle})\right\} \\
& -\frac{c_{9}}{8 M_{0}^{3}} \sigma \cdot \tilde{\mathbf{E}} \times \tilde{\mathbf{E}},
\end{aligned}
$$

where a $\tilde{\triangle}$ denotes the use of improved derivative operators, and $\tilde{E}, \tilde{B}$ are components of the improved field tensor. The heavy-quark propagator was calculated using the evolution quation

$$
\begin{aligned}
G_{t+1}= & \left(1-\frac{H_{0}}{2 n}\right)^{n} U_{4}^{\dagger}\left(1-\frac{H_{0}}{2 n}\right)^{n} \\
& \times(1-\delta H) G_{t} .
\end{aligned}
$$

For $Q \bar{q}$ mesons, we used the standard tadpoleimproved SW action for the light quarks, with 


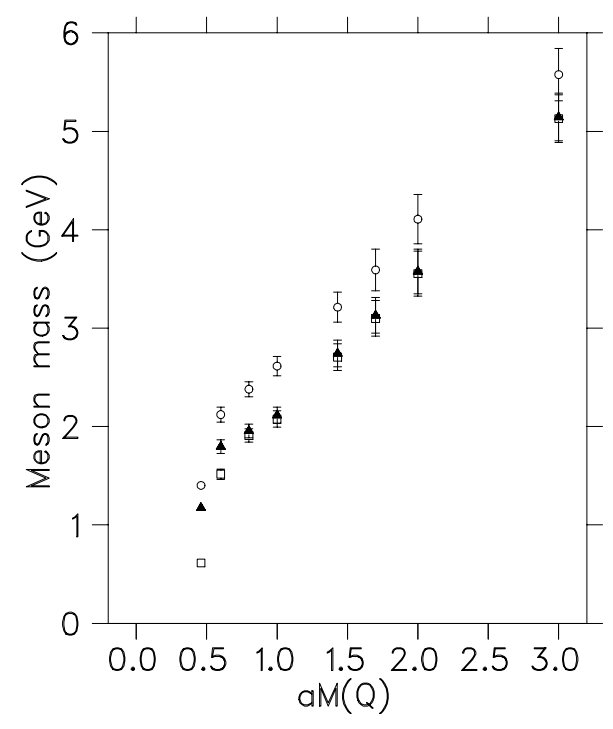

Figure 1. Quarkonium ${ }^{1} S_{0},{ }^{3} S_{1}$ and ${ }^{1} P_{1}$ states as a function of $M_{0}$.

$u_{0}=\langle P l a q\rangle^{-4}$ and $c_{s w}=u_{0}^{-3}$. The light quark $\kappa=0.143$, roughly comparable to the strange quark mass.

Gauge field configurations were created using a standard tadpole- and rectangle-improved action [4]. We used an ensemble of $* * *$ configurations of size $8^{3} \times 24$ at $\beta=6.8$, which gives a tadpole factor of $u_{0}=0.854$.

\section{Quarkonium}

Results for the ${ }^{1} S_{0},{ }^{3} S_{1}$ and ${ }^{1} P_{1}$ states of quarkonium for various values of $M_{0}$ are shown in Figure 1. The stabelisation parameter $n$ in Equation (1) was given values ranging from 2 to 10 depending on the value of $M_{0}$. Meson operators were smeared at source and sink, and their specific forms are detailed in [5].

The ${ }^{1} S_{0}$ states in Figure 1 are found using the kinetic definition of the meson mass,

$E_{\mathbf{p}}=E_{0}+\frac{\mathbf{p}^{2}}{2 a M_{k i n}}$,

where $E_{\mathbf{p}}$ and $E_{0}$ are the simulation energies for

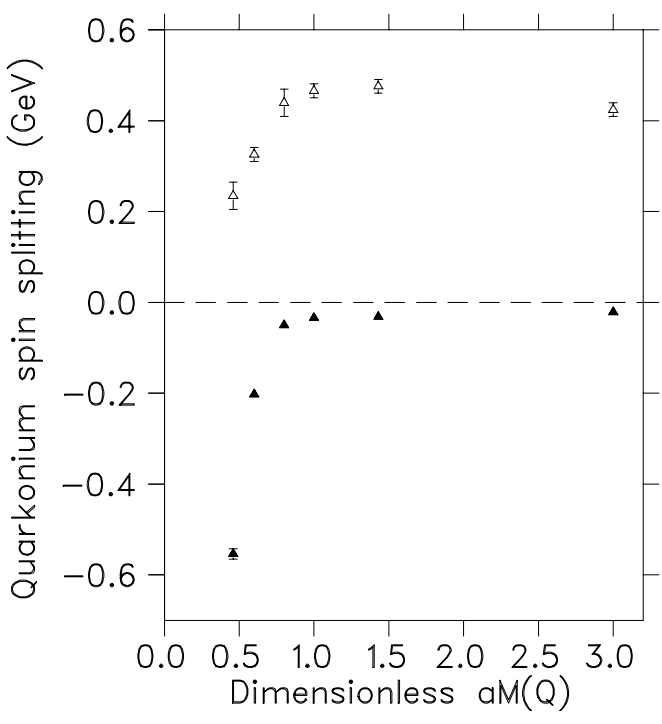

Figure 2. Quarkonium hyperfine and $P-S$ splittings as a function of $M_{0}$.

a meson with finite and zero momentum respectively. This method leads to the large error bars shown on the data, however the general trends are significant here, not the exact energies.

The heaviest $M_{0}$ corresponds to roughly half the bottom quark mass, while the charmonium ground state energy of $\sim 3 \mathrm{GeV}$ corresponds to $a M_{0} \sim 1.5$ to 2 . The lightest $M_{0}$ gives a kinetic mass close to the energy of an $s \bar{s}$ meson, and so we can assume the quarks are well within the relativistic regime at this stage.

Note that the $S$ and $P$ states decrease quite linearly with $M_{0}$, until the bare mass drops significantly below 1 , when they begin to drop quite suddenly. The hyperfine splitting increases significantly at low $M_{0}$, as might be expected from light-meson spectroscopy, but the $S-P$ splitting decreases, against observation. These splittings, with the ${ }^{3} S_{1}$ state taken as the energy zero, are shown in Figure 2.

These results for quarkonium indicate the NRQCD action is indeed beginning to have difficulties at these low bare masses. The situation is worse for the heavy-light spectrum. Figure 3 


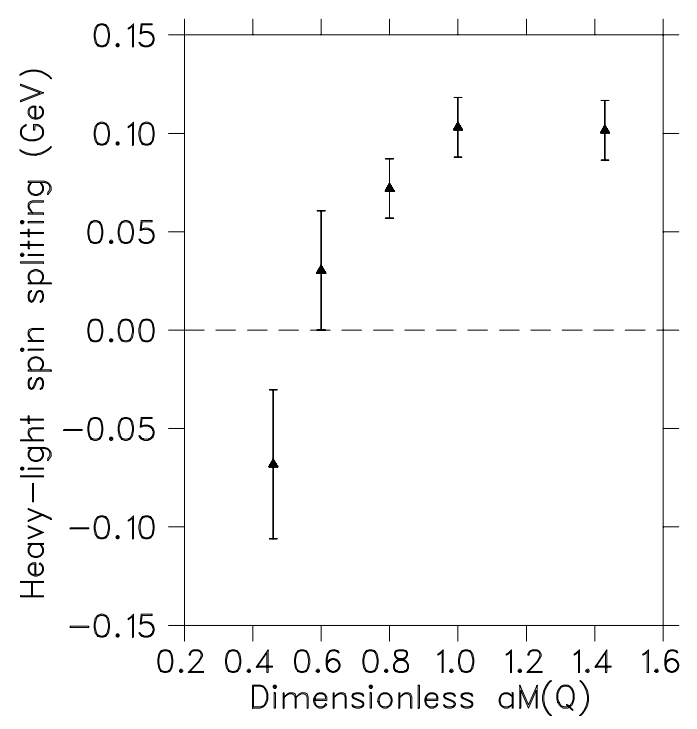

Figure 3. Hyperfine splitting of $Q \bar{q}$ system as a function of $M_{0}$.

shows the hyperfine splittings for the $Q \bar{q}$ system, and note that for the lightest value of $M_{0}$, the splitting is negative - the ${ }^{3} S_{1}$ and ${ }^{1} S_{0}$ energies are in the wrong order. This is obviously a serious pathology.

\section{Discussion}

The results presented here are perhaps not surprising, given that we expect the $M_{0}^{-1}$ dependence of the coefficients in the NRQCD action will ultimately lead to a breakdown as $M_{0}$ decreases. However, we are interested in the exact nature of this breakdown, not to see if NRQCD is a viable alternative for simulating light quarks, but to illuminate any connections between NRQCD and the successes of nonrelativistic quark models.

Lewis and Woloshyn [6] have shown that unphysically large contributions from certain terms in the NRQCD action could be removed by subtracting their expectation values from the Hamiltonian. Their analysis consisted of a thorough systematic examination of the contributions of each correction term in the Hamiltonian. We suspect that the same style of analysis, applied to the low-mass breakdown of NRQCD, would flush out the terms that suffer the worst pathologies, and may even suggest ways they may be strengthened.

One important issue to be aware of, however, is that the coefficients in Equations (1) and (2) are usually given their tree-level value of 1 in NRQCD simulations. For heavy quarks, tadpole improvement of the gauge field links is usually sufficient to account for the most serious corrections beyond tree level. The coefficients $c_{i}$ in Equation (2) will, in general, have non-trivial $\alpha_{s}\left(M_{0}\right)$ dependence, and this will become more important as the quark mass is decreased.

We would like to thank H. Trottier, R. Lewis, S. Collins and J. Heim for their valuable comments. This work is partially funded by the National Science and Engineering Research Council of Canada.

\section{REFERENCES}

1. C.T.H. Davies et al., Phys.Rev. D 50 (1994) 6963-6977.

2. Liu et al., Phys. Rev. D 28 (1983) 170.

3. K. Liu et al., Phys.Rev. D 59 (1999) 112001.

4. See for example N. Shakespeare and H. Trottier, Phys.Rev. D 59 (1999) 014502.

5. H. Trottier, Phys. Rev. D 55 (1997) 68446851.

6. R. Lewis and R. Woloshyn, Phys.Rev. D 58 (1998) 074506. 